Published in final edited form as:

Sex Transm Dis. 2016 January ; 43(1): 57-60. doi:10.1097/OLQ.0000000000000387.

\title{
Field Evaluation of a Dual Rapid Immunodiagnostic Test for HIV and Syphilis infection in Peru
}

\author{
Claire C. Bristow, PhD, MPH, MSc ${ }^{1}$, Segundo R. Leon, MT, MT\&ID 2,3 , Emily Huang, BS ${ }^{4}$, \\ Lourdes B. Ramos, BS ${ }^{2}$, Silver K. Vargas, MPH${ }^{2}$, Juan A. Flores, MSc $^{2}$, Kelika A. Konda, \\ $\mathrm{PhD}^{4}$, Carlos F. Caceres, MD MPH PhD ${ }^{2}$, and Jeffrey D. Klausner, MD MPH ${ }^{4}$ \\ ${ }^{1}$ Division of Global Public Health, Department of Medicine, University of California San Diego, La \\ Jolla, CA, USA \\ 2Unit of Health, Sexuality and Human Development, and Laboratory of Sexual Health, \\ Universidad Peruana Cayetano Heredia, Lima, Peru \\ ${ }^{3}$ Department of Global Health, University of Washington \\ ${ }^{4}$ Program in Global Health, Department of Medicine, University of California Los Angeles, Los \\ Angeles CA, USA
}

\section{Abstract}

Background-Integrated prevention for HIV and syphilis is warranted because both syphilis and HIV infections have evidence-based, scalable interventions using current health care mechanisms. The advent of dual rapid point-of-care tests, single devices that can detect multiple infections using the same specimen, provides the opportunity to integrate the screening of syphilis into HIV programs, potentially increasing the numbers of people tested and allowing for same-day testing and treatment. The aim of this study was to evaluate the MedMira Multiplo Rapid TP/HIV Antibody Test (MedMira Inc, Halifax, Nova Scotia, Canada), a qualitative, rapid immunoassay that detects antibodies to T. pallidum and HIV.

Methods-The reference standard test for comparison to the T. pallidum component of the Multiplo TP/HIV Test was Treponema Pallidum Particle Agglutination assay. For the HIV component, the reference test included a 4th-generation enzyme immunoassay with a confirmatory Western blot test.

Results-The sensitivity and specificity for the HIV antibody component were $93.8 \%$ (95\% CI: $69.8 \%, 99.8 \%$ ) and $100 \%$ (95\% CI: 97.7\%, 100\%), respectively. The Treponema pallidum component of the test had a sensitivity of $81.0 \%$ (95\% CI: $68.1 \%, 94.6 \%)$ and a specificity of $100 \%$ (95\% CI: $97.6 \%, 100 \%)$.

Conclusions-Our study showed excellent performance of the HIV antibody component of the test and very good performance for the Treponema pallidum antibody component of the MedMira Multiplo Rapid TP/HIV Antibody Test, which should be considered to improve screening

Corresponding author information: Claire C. Bristow, PhD MPH MSc, Division of Global Public Health, UCSD, 9500 Gilman Drive 0507, La Jolla, CA 92093-0507, ccbristow@ gmail.com, Phone: 1-415-250-2681.

Conflict of interest statement The authors do not have a commercial or other association that might pose a conflict of interest. 
coverage. Use of effective dual tests will create improved access to more comprehensive care by integrating the screening of syphilis into HIV prevention programs.

\section{Keywords}

Dual test; HIV; syphilis; Treponema pallidum; point-of-care; diagnosis

\section{Introduction}

Syphilis and HIV infection continue to present a substantial global public health burden to groups at high risk of infection such as sex workers, men who have sex with men, migrant populations, pregnant women, and other vulnerable populations that are not likely to access traditional available testing or healthcare services. ${ }^{1,2}$ Those infections share common risk groups and similar modes of transmission, and are often addressed with related strategies. Co-infection is common, and syphilis may increase the risk of HIV acquisition and transmission. ${ }^{3}$ A combined antenatal screening strategy for syphilis and HIV infection, as is recommended by the World Health Organization (WHO) as part of a comprehensive dual elimination initiative, should be implemented to reduce delays in diagnosis and accelerate entrance into treatment. ${ }^{4}$ The advent of dual rapid point-of-care tests, single devices that can detect multiple infections using the same specimen, provides the opportunity to integrate the screening of syphilis into HIV prevention programs, potentially increasing the numbers of people tested and allowing for same-day testing and treatment. 5,6

Results from multiple laboratory and field evaluations support the very good performance of individual syphilis and HIV rapid point-of-care tests. ${ }^{7-14}$ These test devices use specific antigens which form an antigen-antibody complex when specific antibodies are present in the specimen. The binding of the antibody to the antigen contained in the device produces a visible, colored line that is displayed to indicate a positive result. Use of this technology has been critical in the development of dual rapid tests, which enable the simultaneous diagnosis of multiple infections with a single test. Although dual tests for syphilis and HIV infection are relatively new to the point-of-care test market, recent laboratory evaluations have documented high performances for both the Treponema pallidum and HIV components in sera and plasma. ${ }^{15,16}$ However, there are limited data on the actual performance of dual tests in the field.

The MedMira Multiplo Rapid TP/HIV Antibody Test (Medmira Inc, Halifax, Nova Scotia, Canada) is a qualitative, rapid immunoassay that detects antibodies to Treponema pallidum and HIV. The aim of this study was to evaluate the field performance of the MedMira Multiplo Rapid TP/HIV Antibody Test.

\section{Materials and Methods}

\section{Study population and study sites}

Participants were identified at outreach sites in Ventanilla, Callao, a low-income port city adjacent to Lima, Peru. Outreach sites were sites frequented by key populations and included a transgender sex worker house and a beauty salon. Participants were recruited 
through word of mouth and enrolled included those at high risk for HIV and syphilis infections including sex workers, men who have sex with men, and transgender women over the age of 18 and consenting to participate. After obtaining informed consent, a fingerstick whole blood specimen was attained and a venipuncture whole blood specimen was collected from each participant by a trained phlebotomist.

\section{Test under evaluation}

The Multiplo TP/HIV test was conducted on the fingerstick specimen at the site of specimen collection. The Multiplo TP/HIV test is a visually interpreted, immunoassay that qualitatively detects $\operatorname{IgM}$ and $\mathrm{IgG}$ antibodies to recombinant Treponema pallidum antigens (Tp0171 (TpN15), Tp0435 (TpN17) and Tp0574 (TpN47)), and to HIV 1/2 in human serum, plasma, or whole blood. Following application of the specimen, a visible line or dot appears if an antigen-antibody-antigen gold particle complex is formed. The test includes a built-in procedural and reagent control line that should always appear if the specimen has been added to the test cartridge and the test reagents are functioning properly. For this study, incomplete control lines were interpreted as invalid tests. The test was read immediately after the solution was absorbed completely, per the manufacturer's instructions. Two readers interpreted the test results separately. In accordance with standard clinical testing protocols in Peru, participants were given syphilis and HIV test results from reference standard tests within two weeks after enrollment. The Multiplo TP/HIV Test was investigational and therefore the results from the Multiplo TP/HIV were not reported to participants and were not used for clinical management.

\section{Reference standard tests}

Specimens collected by venipucture were transported to the Laboratory of Sexual Health at the Faculty of Sciences of Universidad Peruana Cayetano Heredia for reference testing. The reference laboratory performed serum separation and comparison testing. The reference standard test for comparison to the T. pallidum component of the Multiplo TP/HIV Test was Treponema Pallidum Particle Agglutination (TPPA) (SERODIA-TPPA, Fujirebio Diagnostics, Inc., Japan). In addition, rapid plasma reagin tests (BD Macro-Vue RPR, Beckon-Dickenson, NJ) were performed on all specimens to assist in clinical diagnosis. For the HIV component, the reference test included a 4th-generation enzyme immunoassay (Genscreen ${ }^{\text {TM }}$ ULTRA HIV Ag-Ab, Bio-Rad, France) for the simultaneous qualitative detection of HIV p24 antigen and antibodies to gp41 and gp36 of HIV Type 1 (HIV-1 groups $\mathrm{M}$ and $\mathrm{O}$ ) and HIV Type 2 (HIV-2). All of the specimens that were positive on the enzyme immunoassay underwent a confirmatory Western blot test (NEW LAV BLOT I, Bio-Rad, France).

\section{Data Analysis}

Sensitivity and specificity were estimated and the exact binomial method was used to calculate 95\% confidence intervals (CI). Cohen's Kappa statistic was calculated to assess concordance between the test under evaluation and reference standard tests and to calculate concordance between two test readers. All analyses were conducted using SAS v9.3 (Cary, NC, USA). 


\section{Results}

A total of 205 participants were enrolled in the study, 175 (85.4\%) had valid Multiplo $\mathrm{TP} / \mathrm{HIV}$ tests and were included in the analysis. Of the 175, $16(9.1 \%)$ were confirmed as HIV infected based on the reference standard HIV test protocol. One of the 175 specimens had an indeterminate TPPA result and therefore was excluded from the Treponema antibody detection component of the analysis. Of the 174 specimens with a TPPA test result, 27 (15.5\%) tested positive for presence of treponemal antibodies based on the reference standard TPPA test protocol.

The HIV antibody component of the Multiplo test yielded 15 true positive, 0 false positive, 1 false negative, and 159 true negative results [see Table 1]. The sensitivity and specificity for the HIV antibody component were $93.8 \%$ (95\% CI: 69.8\%, 99.8\%) and 100\% (95\% CI: $97.7 \%, 100 \%)$, respectively. The Kappa coefficient for HIV antibody between the reference and Multiplo results was .97 (95\% CI: .90, 1.00).

The Treponema pallidum antibody component of the test gave 17 true positive, 0 false positive, 4 false negative, and 153 true negative results [see Table 2]. The Treponema pallidum component of the test had a sensitivity of $81.0 \%$ (95\% CI: $58.1 \%, 94.6 \%$ ) and the specificity of $100 \%$ (95\% CI: 97.6\%, 100\%). The Kappa coefficient for concordance of presence of Treponema pallidum antibodies between the reference and evaluation test was . 88 (95\% CI: .77, 1.00). Of the 4 Treponema pallidum false negative results, 1 was rapid plasma reagin reactive.

The Multiplo TP/HIV test results were interpreted separately by two readers. All results for the HIV antibody and the Treponema pallidum antibody detection component were concordant between the two readers (Kappa coefficient: 1.00 (95\% CI: 1.00, 1.00).

\section{Discussion}

We completed a field evaluation of a dual rapid immunodiagnostic test for HIV and syphilis infection. Our study showed excellent performance of the HIV antibody component of the test and very good performance for the Treponema pallidum antibody component. Those performance findings are consistent with field performance evaluations of single rapid tests for HIV and syphilis antibodies ${ }^{7-9,11,14,17}$, and another field study of a dual test ${ }^{18}$. The sensitivity of the Treponema pallidum antibody component of the test was lower than the HIV antibody component. The color indication of a positive Treponema pallidum result was a circle rather than a line which may be harder to identify when the color is faint, which may explain part of of the difference in sensitivity. There are various means to potentially increase the sensitivity of rapid tests that could be further evaluated. One useful mechanism is through the use of an electronic reader that has higher optical detection than the human eye. ${ }^{19}$ This was an immunofiltration assay rather than a lateral flow assay that is the more well-known assay for a rapid test. The Multiplo test can be read immediately after being inoculated with the specimen and reagents without any wait time, a benefit that is not possible with the lateral flow assay. 
Field evaluations are important to identify the performance characteristics of a test, outside the ideal conditions of a laboratory setting, where the tests will be used in practice. Rapid point-of-care tests can result in same-day treatment for syphilis and improved linkage to care for HIV infection. In addition, point-of-care tests can be used outside of clinical settings and in places with limited laboratory access to improved screening coverage. ${ }^{7}$ Multiplex rapid tests provide additional advantages by requiring less equipment and supplies, simplified procurement and supply chain management, and require only one specimen to test for multiple infections. Furthermore, patients may also prefer streamlined specimen collection and testing for multiple infections. ${ }^{20}$

This study was subject to some limitations. Firstly, almost $15 \%$ of the Multiplo TP/HIV tests gave invalid control lines. Further research should be considered to find ways to improve test controls. However, having a control mechanism within the test allowed us to exclude specimens that may have been inadequately added to the test cartridge or that contained test reagents that were not functioning properly. Another limitation is that findings on the test performance in this setting may vary in other settings and therefore this study should be repeated in other sites to assess generalizability. Additionally, althrough field evaluations are essential to understand how a test would perform once rolled out, this study was performed by motivated study staff that may not be representative of the clinical staff that would be responsible for screening. Further research is warranted to determine how a dual test would perform and can be integrated into the flow of a busy sexual health clinic. Another limitation of this study is that we only used one treponemal reference test, which displays high performance but may be an imperfect reference standard test. We did, however, have access to rapid plasma reagin results which we used to provide some explanation on discordant Treponema pallidum antibody test results. A strength of this study is that we used a $4^{\text {th }}$ generation HIV antigen-antibody reference test; the $4^{\text {th }}$ generation tests are the most specific and sensitive tests on the market. Most rapid tests for detection of syphilis, including the Multiplo TP/HIV test, detect treponemal antibodies which can persist for life even following curative treatment. In settings with a high prevalence of syphilis, a confirmatory nontreponemal test, such as the rapid plasma reagin, may be necessary.

HIV and syphilis have similar risk groups, can both be sexually and congenitally transmitted, and are associated with adverse pregnancy outcomes. The World Health Organization has called for the elimination of mother-to-child of HIV and syphilis infection through a harmonized approach for the improvement of health outcomes for mothers and children. ${ }^{4}$ Key populations at risk for HIV and syphilis include sex workers, men who have sex with men, migrant populations, pregnant women, and other vulnerable populations that are not likely to access traditional available testing or healthcare services. ${ }^{21,22}$ These populations often also face substantial barriers to accessing and utilizing traditional health care services and would greatly benefit from the use of rapid point-of-care tests. This study recruited participants from these key populations in Lima, Peru to understand how dual testing performs in a population where dual testing may be useful. Implementing an accurate, simple, and affordable dual screening strategy for HIV and syphilis could improve test uptake and accessibility of testing to reduce time to treatment. ${ }^{6}$ The MedMira Multiplo 
Rapid TP/HIV Antibody Test should be considered for implementation in settings where HIV and syphilis testing are needed.

\section{Acknowledgments}

Sources of support This study was funded through though an NIH/NIAID R-01 study \#1R01AI099727-01. All test materials were donated by Medmira Inc.

\section{REFERENCES}

1. Newman L. Research TCiSaRH. Dual elimination of mother-to-child transmission (MTCT) of HIV and syphilis. 2013 http://wwwgfmerch/SRH-Course-2013/sti/pdf/Dual-EMTCT-HIV-syphilisNewman-2013pdf;

2. UNAIDS. [accessed September 18, 2014] Global report: UNAIDS report on the global AIDS epidemic 2013. 2013. http://wwwunaidsorg/en/media/unaids/contentassets/documents/ epidemiology/2013/gr2013/UNAIDS_Global_Report_2013_enpdf

3. Sanchez J, Lama JR, Peinado J, et al. High HIV and ulcerative sexually transmitted infection incidence estimates among men who have sex with men in Peru: awaiting for an effective preventive intervention. Journal of acquired immune deficiency syndromes. 2009; 51(Suppl 1):S47S51. [PubMed: 19384102]

4. WHO. Elimination of mother-to-child transmission (EMTCT) of HIV and syphilis: Global guidance on criteria and processes for validation. 2014 Available at: http://wwwwhoint/reproductivehealth/ publications/rtis/9789241505888/en/.

5. Dinh TH, Kamb ML, Msimang V, et al. Integration of preventing mother-to-child transmission of HIV and syphilis testing and treatment in antenatal care services in the Northern Cape and Gauteng provinces, South Africa. Sexually transmitted diseases. 2013; 40(11):846-851. [PubMed: 24113405]

6. Klausner JD. The sound of silence: missing the opportunity to save lives at birth. Bulletin of the World Health Organization. 2013; 91(3):158-A. [PubMed: 23476083]

7. Benzaken AS, Sabido M, Galban E, et al. Field performance of a rapid point-of-care diagnostic test for antenatal syphilis screening in the Amazon region, Brazil. International journal of STD \& AIDS. 2011; 22(1):15-18. [PubMed: 21364061]

8. Bergman J, Gratrix J, Plitt S, et al. Feasibility and Field Performance of a Simultaneous Syphilis and HIV Point-of-Care Test Based Screening Strategy in at Risk Populations in Edmonton, Canada. AIDS research and treatment. 2013; 2013:819593. [PubMed: 24527210]

9. Kagulire SC, Opendi P, Stamper PD, et al. Field evaluation of five rapid diagnostic tests for screening of HIV-1 infections in rural Rakai, Uganda. International journal of STD \& AIDS. 2011; 22(6):308-309. [PubMed: 21680664]

10. Montoya PJ, Lukehart SA, Brentlinger PE, et al. Comparison of the diagnostic accuracy of a rapid immunochromatographic test and the rapid plasma reagin test for antenatal syphilis screening in Mozambique. Bulletin of the World Health Organization. 2006; 84(2):97-104. [PubMed: 16501726]

11. Nessa K, Alam A, Chawdhury FA, et al. Field evaluation of simple rapid tests in the diagnosis of syphilis. International journal of STD \& AIDS. 2008; 19(5):316-320. [PubMed: 18482961]

12. Tucker JD, Bu J, Brown LB, Yin YP, Chen XS, Cohen MS. Accelerating worldwide syphilis screening through rapid testing: a systematic review. The Lancet infectious diseases. 2010; 10(6): 381-386. [PubMed: 20510278]

13. Tucker JD, Bien CH, Peeling RW. Point-of-care testing for sexually transmitted infections: recent advances and implications for disease control. Curr Opin Infect Dis. 2013; 26(1):73-79. [PubMed: 23242343]

14. West B, Walraven G, Morison L, Brouwers J, Bailey R. Performance of the rapid plasma reagin and the rapid syphilis screening tests in the diagnosis of syphilis in field conditions in rural Africa. Sexually transmitted infections. 2002; 78(4):282-285. [PubMed: 12181468] 
15. Bristow CC, Adu-Sarkodie Y, Ondondo RO, et al. Multi-site laboratory evaluation of a dual HIV/ syphilis point-of-care rapid test for simultaneous detection of HIV and syphilis. Open Forum Infectious Diseases. 2014; 1(1)

16. Esfandiari, J.; Gunasekera, D.; Sayegh, F., et al. Performance Evaluation of the Chembio DPP ${ }^{\circledR}$ HIV-Syphilis Assay: A Novel, Point-of-care Rapid HIV $1 / 2$ and Syphilis. Abstract presented at, 2012 HIV Diagnostics Conference 2012; 12 December, 2012; Atlanta, GA. (https:// custom.cvent.com/ADE0EB81B3184D618E2FB8340F1EC28E/files/ 29f3717707a44f91859f65feb4cefec6.pdf)

17. Strasser S, Bitarakwate E, Gill M, et al. Introduction of rapid syphilis testing within prevention of mother-to-child transmission of HIV programs in Uganda and Zambia: a field acceptability and feasibility study. Journal of acquired immune deficiency syndromes. 2012; 61(3):e40-e46. [PubMed: 22820810]

18. Bristow, CC.; Leon, S.; Ramos Cordova, L., et al. Clinical evaluation of a dual rapid diagnostic test for HIV and syphilis, SD Bioline HIV/syphilis Duo, Lima, Peru. Atlanta, GA: CDC STD Prevention Converence 2014; 2014. http://www.cdc.gov/stdconference/2014/2014-std-preventionconference-abstracts.pdf, editor

19. Mudanyali O, Dimitrov S, Sikora U, Padmanabhan S, Navruz I, Ozcan A. Integrated rapiddiagnostic-test reader platform on a cellphone. Lab on a chip. 2012; 12(15):2678-2686. [PubMed: 22596243]

20. Bristow, CC.; Lee, SJ.; Leon, S., et al. Assessing factors to increase uptake of testing for syphilis and HIV in men who have sex with men and transgender women in Lima, Peru 2013. Atlanta, GA: CDC STD Prevention Conference; 2014. http://www.cdc.gov/stdconference/2014/2014-stdprevention-conference-abstracts.pdf

21. Gardner EM, McLees MP, Steiner JF, Del Rio C, Burman WJ. The spectrum of engagement in HIV care and its relevance to test-and-treat strategies for prevention of HIV infection. Clinical infectious diseases : an official publication of the Infectious Diseases Society of America. 2011; 52(6):793-800. [PubMed: 21367734]

22. Geibel S, Tun W, Tapsoba P, Kellerman S. HIV vulnerability of men who have sex with men in developing countries: Horizons studies, 2001-2008. Public Health Rep. 2010; 125(2):316-324. [PubMed: 20297760] 


\section{Summary}

We evaluated MedMira Multiplo Rapid TP/HIV Antibody Test for the simultaneous detection of HIV and syphilis infection. The dual test shows good performance in a field setting in Lima, Peru. 


\section{Table 1}

Field performance for detection of HIV antibodies using a dual HIV/syphilis test (N=205).

\begin{tabular}{|c|c|c|c|c|c|c|c|}
\hline & & \multicolumn{2}{|c|}{$\underline{\text { Number of samples }}$} & \multirow[t]{2}{*}{ Total } & $\begin{array}{l}\text { Sensitivity } \\
\text { (95\% CI) }\end{array}$ & $\begin{array}{l}\text { Specificity } \\
(95 \% \text { CI })\end{array}$ & $\begin{array}{c}\text { Kappa Coefficient } \\
(95 \% \text { CI })\end{array}$ \\
\hline & & Ref test + & Ref test - & & $93.8 \%$ & $100 \%$ & .97 \\
\hline & Multiplo Test + & 15 & 0 & 15 & $(69.8 \%, 99.8 \%)$ & $(97.7 \%, 100 \%)$ & $(.90,1.00)$ \\
\hline & Multiplo Test - & 1 & 159 & 160 & & & \\
\hline Total & & 16 & 159 & 175 & & & \\
\hline
\end{tabular}

Reference testing was conducted with an HIV enzyme immunoassay with a western blot for confirmation of positive result. Thirty tests gave an invalid control line and were excluded from analysis 


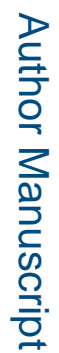

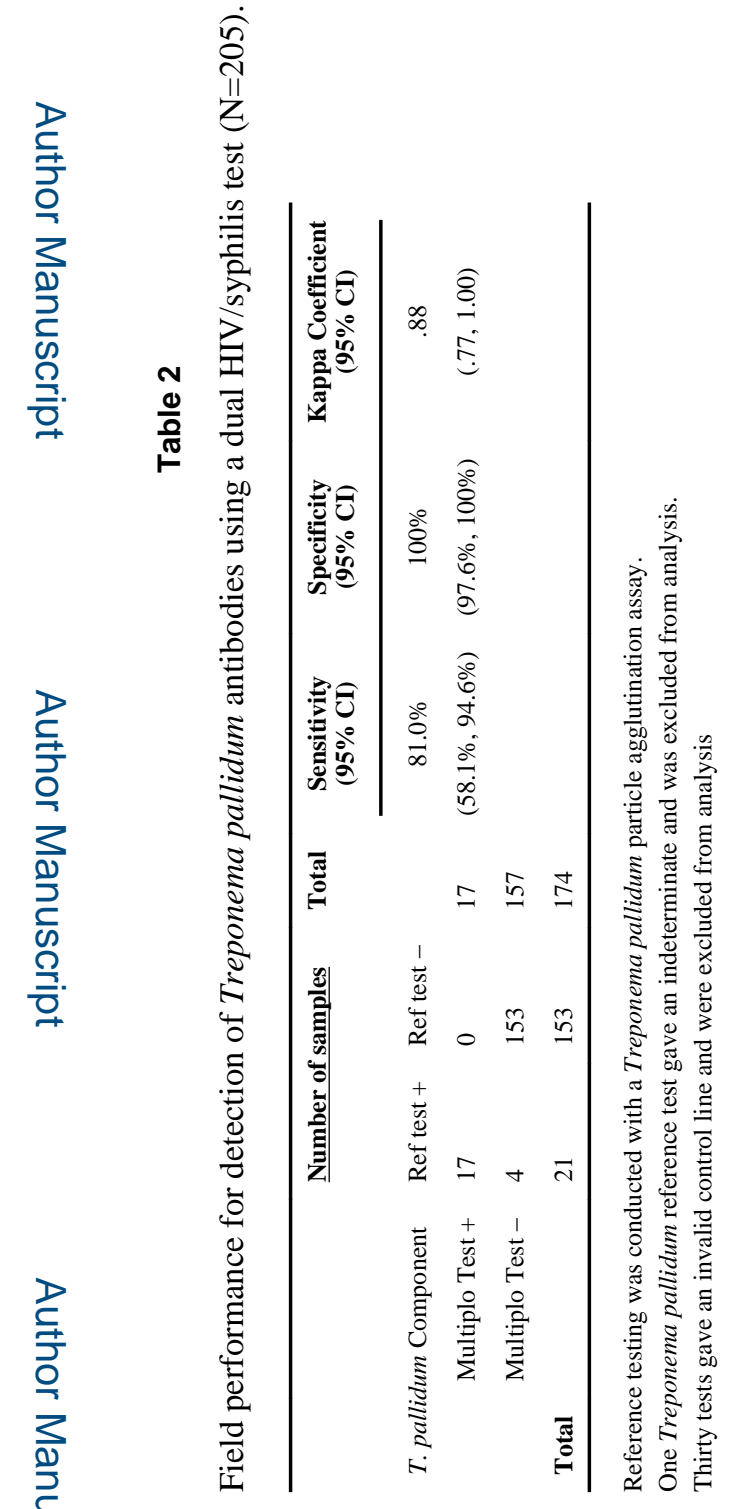

Sex Transm Dis. Author manuscript; available in PMC 2017 January 01. 